\title{
Pleural effusion after microtia reconstructive surgery -A case report-
}

\author{
Yun Hee Kim ${ }^{1}$, Jin Namkung ${ }^{1}$, Byung Gun Lim², Sam Hong Min ${ }^{1}$, Hye Won Shin ${ }^{1}$, and Choon Hak Lim ${ }^{1}$ \\ Department of Anesthesiology and Pain Medicine, ${ }^{1}$ College of Medicine, Korea University, ${ }^{2}$ Korea University Guro Hospital, Seoul, \\ Korea
}

Microtia reconstructive surgery is usually a multi-stage repair procedure that involves the use of cartilage and skin grafts. Complications can arise at both ear reconstruction sites and cartilage donor sites. In particular, pneumothorax, atelectasis, chest scars, and chest deformities are known to be associated with the harvesting of costal cartilage. However, delayed pleural effusion can also develop. Our patient complained of a cough and chest pain at 5 days postoperatively, and pleural effusion was detected by chest radiography. However, thoracentesis was not performed and the effusion resolved spontaneously and completely. (Korean J Anesthesiol 2011; 61: 166-168)

Key Words: Cartilage harvest, Microtia, Pleural effusion.

Microtia is a congenital abnormality involving the near absence of the external ear, and thus, microtia surgery requires total auricular reconstruction. The primary reconstruction technique usually employs the patient's rib cartilage, but complications can arise at ear reconstruction and donor sites. Pneumothorax, atelectasis, chest scars, and chest deformities are also known to be associated with the harvesting of costal cartilage [1,2].

Here, the authors report a case of pleural effusion after microtia reconstruction with no significant intraoperative damage.

\section{Case Report}

A 12-year-old (150 cm, $48 \mathrm{~kg}$ ) female was scheduled for microtia reconstructive surgery. Preoperative laboratory findings and her chest radiograph were all normal. She was premedicated with midazolam $2 \mathrm{mg}$ and atropine $0.5 \mathrm{mg} \mathrm{IM}$, and general anesthesia was induced with propofol $100 \mathrm{mg}$ and rocuronium $50 \mathrm{mg}$ and maintained with oxygen $(2 \mathrm{~L} / \mathrm{min}), \mathrm{N}_{2} \mathrm{O}$ (2 L/min), and sevoflurane (2.0-3.0 vol\%).

Costal cartilage was harvested from the left $6,7,8$, and $9^{\text {th }}$ ribs and modeled to form an auricle, which was then implanted at the reconstruction site. The cartilage donor site was soaked with saline and then the Valsalva maneuver was performed to detect air bubbles to exclude pneumothorax. No air bubbles were detected. After surgery, intravenous patient-controlled analgesia with fentanyl and ketorolac was administered for postoperative pain management until the $3^{\text {rd }}$ postoperative day. In addition, analgesics were administered as needed from the

Received: October 4, 2010. Revised: February 15, 2011. Accepted: February 16, 2011.

Corresponding author: Choon Hak Lim, M.D., Ph.D., Department of Anesthesiology and Pain Medicine, College of Medicine, Korea University, Anam-dong 5-ga, Sungbuk-gu, Seoul 136-705, Korea. Tel: 82-2-920-5632, Fax: 82-2-929-2936, E-mail: yourejoice@korea.ac.kr

(c) This is an open-access article distributed under the terms of the Creative Commons Attribution Non-Commercial License (http:// creativecommons.org/licenses/by-nc/3.0/), which permits unrestricted non-commercial use, distribution, and reproduction in any medium, provided the original work is properly cited. 
$4^{\text {th }}$ postoperative day because she complained about ongoing pain in the surgical area that interfered with sleep. However, the pain management was less than effective and anxiety and pain levels were relatively high (her VAS score at the time was 6).

On the $5^{\text {th }}$ postoperative day, the patient complained of a cough and mild chest discomfort, which was increased by deep inspiration on the left side. However, she was afebrile and her vital signs were normal. Chest radiography performed at the time revealed a small left-sided pleural effusion (Fig. 1). Thoracentesis was not indicated emergently, and she was managed conservatively.

On the $9^{\text {th }}$ postoperative day, her symptoms were relieved and chest radiography showed complete resolution of the effusion with minimal pleural thickening on the left side (Fig. 2). She was discharged on the $14^{\text {th }}$ post-operative day in a stable condition.

\section{Discussion}

Microtia, a small and abnormal auricle, affects in approximately 1 live birth per $7,000-8,000$, occurring more often in right ears (a right-to-left ratio of $3: 2$ ), males ( $\mathrm{M}: \mathrm{F}$ ratio
$2.5: 1$ ), and unilateral cases (a unilateral-to-bilateral ratio of $4: 1$ ) [3]. Microtia reconstructive surgery is usually a multistage procedure involving cartilage and skin grafts, and thus, complications can arise at both ear reconstruction and donor sites. The principal auricular complications are necrosis of skin overlying the graft site and protrusion of donor cartilage. In addition, its perioperative pulmonary complications include intraoperative pleural perforations $(22 \%)$ and postoperative atelectasis without evidence of pneumothorax (8\%) [4]. Pulmonary complications are a major cause of morbidity and mortality during the perioperative period.

Generally, pleural effusions are caused by systemic or local factors that alter the pleural fluid production/absorption balance due to an elevated hydrostatic pressure (e.g., congestive heart failure), decreased oncotic pressure (e.g., hypoalbuminemia), increased negative pressure in the pleural space (e.g., large atelectasis), or increased permeability of the microvascular circulation due to inflammatory mediators (e.g., pneumonia) [5,6]. Pleural effusions may also occur due to impaired lymphatic drainage and increased diaphragm permeability due to surgical interventions [7-11]. Our patient
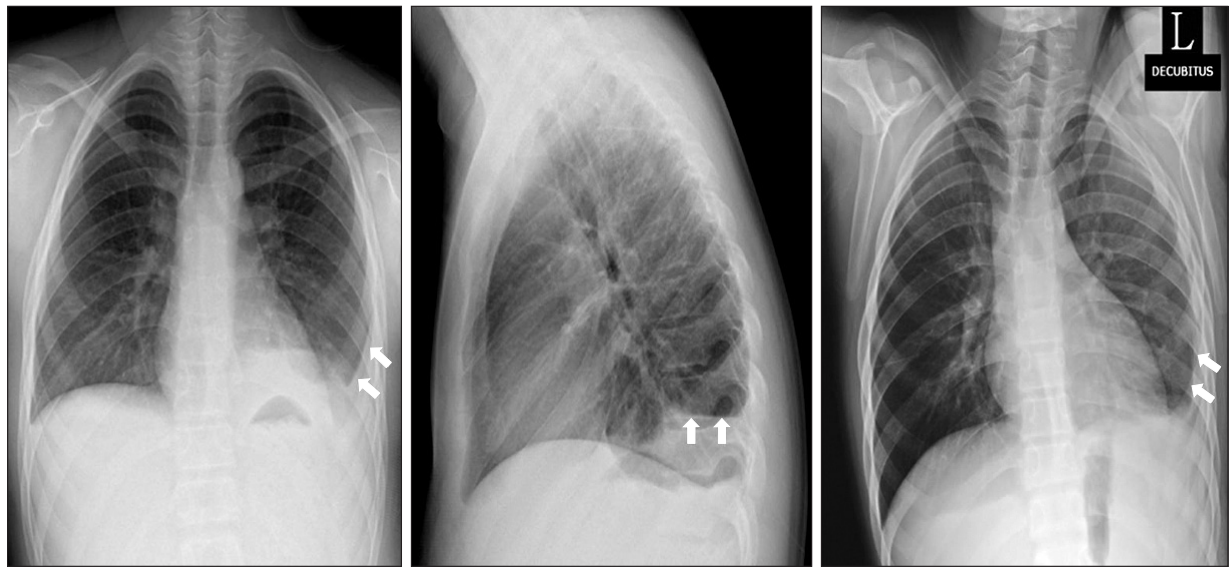

Fig. 1. On the $5^{\text {th }}$ postoperative day, left costophrenic angle blunting was observed on chest PA, lateral and left decubitus views (left, center, right, respectively).
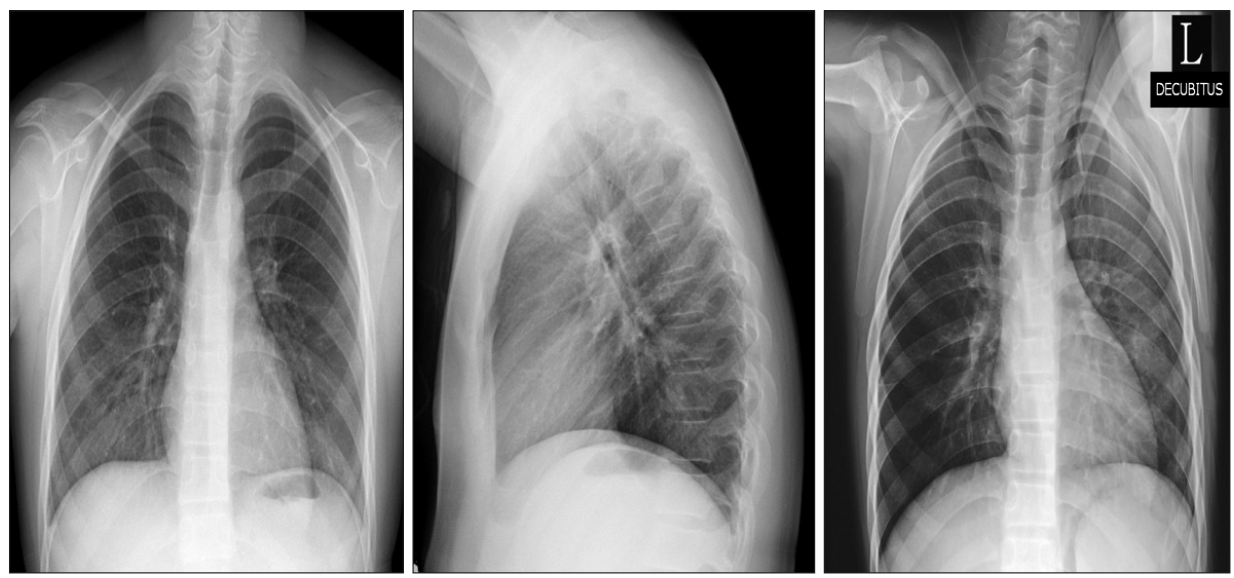

Fig. 2. On the $9^{\text {th }}$ postoperative day, complete resolution of the left pleural effusion was evident by chest PA, lateral and left decubitus views (left, center, right, respectively). 
had unilateral pleural effusion at cartilage donor sites, and thus, we speculate that local factors were responsible for the pleural effusion. First, surgical trauma to the ipsilateral parietal pleura on the cartilage donor site might have caused the pleural effusion. During the procedure, pneumothorax was detected by the presence of air bubbles when cartilage donor sites were soaked with saline. However, no method is available to detect tiny parietal pleural defects. Second, irritation of the pleura during surgery could lead to increase permeability of the microvascular circulation and induce pleural effusion, and the slow accumulation of pleural fluid could induce delayed postoperative pleural effusion. Third, postoperative pain and discomfort may have caused atelectasis and negative pressure in the pleural space might then have induced pleural effusion. Uppal et al. [1] reported that complaints of pain peaked at around one week after microtia surgery and then diminished slowly over 3 months. Pain management was less than effective in our patient. Nevertheless, significant atelectasis was not shown on her chest radiograph.

Although almost all cases should be evaluated by diagnostic thoracentesis, this should not be done when the effusion is too small to be safely aspirated $(<10 \mathrm{~mm}$ thick by ultrasonography or lateral decubitus radiography) or when the effusion can be explained by underlying congestive heart failure, recent thoracic or abdominal surgery, or postpartum surgery [6]. In our patient, thoracentesis was not performed because of a small amount of effusion and the patient was carefully monitored instead. However, a large effusion may produce dyspnea, coughing, pain, or pyrexia and places the patient at high risk of infection. Furthermore, the additional suffering interferes with postoperative recovery, prolongs hospital stays, and increases cost.

Our case suggests that although general anesthesia and the operation are uneventful, pleural effusion may occur. So, close attention is required during the first week after microtia reconstructive surgery. Standard posteroanterior and lateral chest radiography remains the most important means for initially diagnosing pleural effusion, but at least $200 \mathrm{ml}$ of fluid must accumulate before it becomes visible on posteroanterior radiographs, while as little as $5 \mathrm{ml}$ of pleural fluid can be seen in lateral decubitus view [12], which suggests that this view should be used for routine postoperative testing. Furthermore, postoperative pain should be managed effectively for at least 1 week after surgery.

\section{References}

1. Uppal RS, Sabbagh W, Chana J, Gault DT. Donor-site morbidity after autologous costal cartilage harvest in ear reconstruction and approaches to reducing donor-site contour deformity. Plast Reconstr Surg 2008; 121: 1949-55.

2. Ohara K, Nakamura K, Ohta E. Chest wall deformities and thoracic scoliosis after costal cartilage graft harvesting. Plast Reconstr Surg 1997; 99: 1030-6.

3. Aguilar EF. Auricular reconstruction in congenital anomalies of the ear. Facial Plast Surg Clin North Am 2001; 9: 159-69.

4. Thomson HG, Kim TY, Ein SH. Residual problems in chest donor sites after microtia reconstruction: a long term study. Plast Reconstr Surg 1995; 95: 961-8.

5. Chu KJ, Yao XP, Fu XH. Factors related to pleural effusion following hepatectomy for primary liver cancer. Hepatobiliary Pancreat Dis Int 2007; 6: 58-62.

6. Yataco JC, Dweik RA. Pleural effusions: evaluation and management. Cleve Clin J Med 2005; 72: 854-6.

7. Labidi M, Baillot R, Dionne B, Lacasse Y, Maltais F, Boulet LP. Pleural effusions following cardiac surgery. Chest 2009; 136: 160411.

8. Nielsen PH, Jepsen SB, Olsen AD. Postoperative pleural effusion following upper abdominal surgery. Chest 1989; 96: 1133-5.

9. Bacon BR, Bailey-Newton RS, Connors AF Jr. Pleural effusions after endoscopic variceal sclerotherapy. Gastroenterology 1985; 88: 19104.

10. Spizarny DL, Gross BH, McLoud T. Enlarging pleural effusion after liver transplantation. J Thorac Imaging 1993; 8: 85-7.

11. Shitrit D, Izbicki G, Fink G, Bendayan D, Aravot D, Saute M, et al. Late postoperative pleural effusion following lung transplantation: characteristics and clinical implications. Eur J Cardiothorac Surg 2003; 23: 494-6.

12. Gallardo X, Castaner E, Mata JM. Benign pleural diseases. Eur J Radiol 2000; 34: 87-97. 